\title{
Arterial Pulse Waveform Characteristics Difference between the Three Trimesters of Healthy Pregnant Women
}

\author{
Kunyan Li, Song Zhang, Zhenyu Chi, Yimin Yang*, Hongqing Jiang, Lin Yang, Anran Wang, Lei Zhang, \\ Fei Chen, Dingchang Zheng
}

\begin{abstract}
During pregnancy, the pregnant mother undergoes significant physiological changes in order to accommodate the developing fetus. In recent years, arterial pulse wave has been widely used to reflect these physiological changes. The aim of this study was to investigate the changes of radial pulse and photoplethysmography (PPG) pulse waveform characteristic with gestational age in normal pregnant women. 40 pregnant women volunteers were recruited from February 2016 to September 2016 from the Haidian Maternal \& Child Health Hospital in Beijing. Both radial pulses and PPG pulses were recorded simultaneously using a PowerLab data collection system at a sampling rate of $1000 \mathrm{~Hz}$ for offline analysis. Their pulses were measured from each pregnant woman at three trimesters (first trimester between week 11-13; second trimester between week $20-22$ and the third trimester between week 37-39). Three waveform characteristics (total pulse area; pulse area1: the area before the notch position; pulse area2: the area after the notch position) were derived. The results showed that the total pulse area and pulse area2 from both radial and PPG pulses decreased significantly between two paired consecutive trimesters (all $\mathbf{P}<0.01$, except the comparisons between the second and third trimesters for PPG pulses). In summary, this study has quantified the pulse waveform characteristic differences in terms of pulse areas between the three trimesters, providing useful scientific evidence to better understand the cardiovascular physiological changes during normal pregnancy.
\end{abstract}

\section{INTRODUCTION}

During pregnancy, the pregnant mother undergoes significant physiological changes in order to nurture and accommodate the developing fetus. These changes begin after conception and affect different organ systems in the body, including cardiovascular, hematologic, metabolic, renal, and respiratory systems [1]. Increased blood sugar, breathing, and cardiac output are some of the expected changes that allow a pregnant woman's body to facilitate the growth and development of the fetus during pregnancy. For most women with normal pregnancy, these changes reverse after delivery with minimal residual effects. It is therefore physiologically

Kunyan Li, Song Zhang, Zhenyu Chi, Yimin Yang, Lin Yang, Anran Wang, Lei Zhang are with Beijing University of Technology, Beijing, China (e-mail:likunyan_77@qq.com, zhangsong@bjut.edu.cn, chizhenyu@emails.bjut.edu.cn, yym@bjut.edu.cn, yanglin@bjut.edu.cn, wanganran@emails.bjut.edu.cn, zl@beijingyes.com. *Telephone: 008613426181228; fax: 010-67392010; e-mail: yanglin@ bjut.edu.cn).

Hongqing Jiang is with Haidian Maternal \& Child Health Hospital (e-mail: drjhq91@ sohu.com).

Dingchang Zheng is with Department of Medical Science and Public Health, Faculty of Medical Science, Anglia Ruskin University, Chelmsford CM1 1SQ, UK (e-mail: dingchang.zheng@anglia.ac.uk)

Fei Chen is with Department of Electrical and Electronic Engineering, Southern University of Science and Technology, Shenzhen, China (e-mail: fchen@sustc.edu.cn) important to understand the normal physiological changes during normal pregnancy as this may help identify abnormal changes.

It is well accepted that arterial pulse waveform contains extremely useful information in association with physiological and pathological changes of cardiovascular system. The waveform shape, including the rhythms, the amplitude of the wave and the speed of how fast the arterial pulse travels, has been used to reflect the physiological and pathological changes of the human cardiovascular system [2-3]. In practice, arterial pulses are often obtained from the wrist using pressure sensor (radial pulses) or from the fingertip using optical sensors (PPG pulses). Pulse wave analysis is a non-invasive, cheap method. It has been widely used to detect changes of physiological characteristics clinically. Specifically, analysis of both the radial and PPG pulse waveform characteristics is an effective method to evaluate the cardiovascular function [4-5]. Some published studies employed pulse wave shape analysis to assess mechanical aspects of arterial aging [6]. The total arterial pulse area and the areas before and after the notch position have been investigated by Wang et al to investigate their changes during exercise and recovery [7], and concluded that these waveform characteristics are useful in better understanding the physiological changes.

In recent decades, some researchers have attempted to understand pulse wave change during pregnancy in association with hemodynamic changes. The brachial-ankle pulse wave velocity has been used to evaluate the arterial stiffness during pregnancy on postpartum [8]. To the best of our knowledge, the changes of total pulse area, the pulse areas before and after the dicrotic notch position have not been comprehensively investigated during pregnancy. In addition, these waveform characteristic derived from the PPG pulse have not been compared with the corresponding changes from the radial pulses during pregnancy.

The aims of this study were to quantify the difference of arterial pulse waveform characteristics between the three trimesters in normal pregnancy women, and compare their differences between the waveform characteristics derived from PPG and radial pulses.

\section{METHODS}

\section{A. Subject information}

In this study, 40 pregnant women volunteers were recruited between February 2016 and September 2016 at the Haidian Maternal \& Child Health Hospital, Beijing. Each of the participants understood the purpose of the study. The Institution's Ethical Review Board approved all experimental procedures involving human subjects. The research received 
the ethics approval from the Ethics Committee of Beijing University of Technology. All experiments were performed in accordance with the Declaration of Helsinki. The participants signed the consent from before the formal test. All pregnant women met the following inclusion criteria: (1) single pregnancy; (2) the normal menstrual cycle; (3) no chronic hypertension, diabetes, anemia, and other cardiovascular disease during pregnancy, and liver and kidney function is normal; (4) not taking blood pressure drugs. All the basic clinical information of the recruited pregnant women, including their age, height, weight and the body index (BMI), is shown in Table 1.

TAB.1 BASIC INFORMATION OF NORMAL PREGNANT WOMEN

\begin{tabular}{llll}
\hline Age (year) & Height $(\mathrm{m})$ & Weight $(\mathrm{kg})$ & $\mathrm{BMI}^{\mathrm{a}}$ \\
\hline $31.03 \pm 3.43$ & $1.62 \pm 0.05$ & $56.61 \pm 10.77$ & $21.81 \pm 3.03$ \\
\hline
\end{tabular}

\section{B. Arterial pulse measurement procedure}

The arterial pulse measurements were performed in a quiet clinical measurement room at the Haidian Maternal \& Child Health Hospital, China. All the pregnant women were asked to sit quietly for 5 minutes to achieve stable heart rate. Resting blood pressure and heart rate were measured from each individual using a validated electronic sphygmomanometer (HEM-7124 from Omron Crop.) before the simultaneous measurements of the radial pulse and PPG pulses. A pressure sensor was placed on the left wrist to record the radial pulses for 3 minutes using a PowerLab data collection system (ADInstrumrnts Pty Ltd., PowerLab 8/35, Bella Vista NSW 2153, Australia) at a sampling rate of $1000 \mathrm{~Hz}$. Simultaneously, PPG pulses were recorded from an optical sensor placed on the left index finger.

For each pregnant woman, three visits were required for three pulse measurements at the three trimesters (first trimester between week 11-13; second trimester between week 20-22; third trimester between week 37-39).

\section{Pulse waveform characteristics determination}

All the recorded radial pulses and PPG pulses from each measurement were firstly processed to remove baseline drift. They were then normalized to get normalized radial pulse waveform and normalized PPG pulse waveform, as shown in Fig.1. First and second derivatives of the normalized waveform was analyzed to find the notch point ( $\mathrm{N}$ point, as show in fig.1), corresponding to the maximum point after the first zero-crossing point in first derivative. The detection of notch point was also manually checked. Finally, from the normalized pulse waveforms, three waveform characteristics were extracted, including the 'total Pulse Area' under the waveform (which was computed from the normalized pulse waveform as: Total Area $=\int_{0}^{100} Y(t) d t$ ), 'Pulse Area1' (computed as Pulse Area1 $=\int_{0}^{N} Y(t) d t$ ) and 'Pulse Area2' (Area2 $\left.=\int_{N}^{100} Y(t) d t\right)$. $\mathrm{Y}(\mathrm{t})$ is the waveform of radial and PPG pulses, t range from 0 to 100 .

\section{Statistical analysis}

The mean \pm SD of all the basic clinical parameters (heart rate, blood pressure) and the pulse waveform characteristics (PPG total pulse area, PPG pulse area1, PPG pulse area2, radial total pulse area, radial pulse area1, and radial pulse area2) were calculated across all the pregnant women separately for the PPG and radial pulses, and for the three trimesters. Analysis of variance after adjusting for blood pressure and post hoc multiple comparisons were then performed using SPSS to compare whether there were significant differences in these arterial waveform characteristics between the three trimesters. A p $<0.05$ was used as the significant criterion for all the parameters.

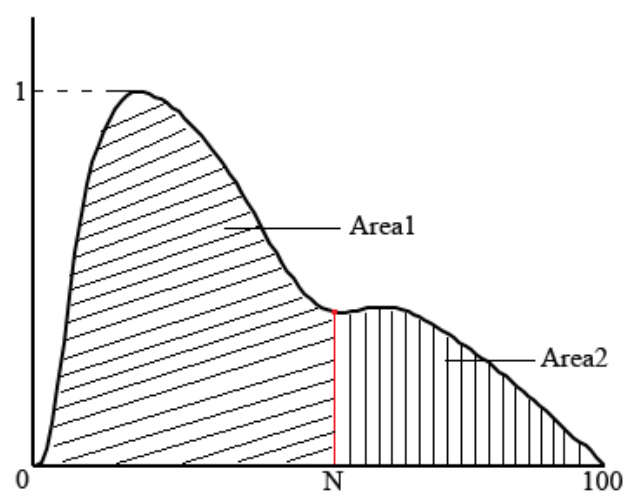

Fig.1. Normalised pulse waveform and the definitions of total pulse area, pulse area 1 and pulse area 2 .

\section{RESUltS}

\section{A. Heart rate and blood pressure difference between the three trimesters}

As show in Table 2, the average heart rate increased significantly from $81 \pm 11$ beats/min at the first trimester to $92 \pm 13$ beats/min at the third trimester of pregnancy $(\mathrm{P}<0.05)$. The corresponding changes of blood pressure were $114 \pm 10$ $\mathrm{mmHg}$ vs $109 \pm 11 \mathrm{mmHg}$ for $\mathrm{SBP}(\mathrm{P}=0.01)$, and $72 \pm 10$ $\mathrm{mmHg}$ vs $69 \pm 10 \mathrm{mmHg}$ for $\mathrm{DBP}(\mathrm{P}=0.12)$.

TAB. 2 Heart Rate And Blood Pressures Of Pregnant Women At the THREE TRIMESTERS.

\begin{tabular}{llll}
\hline & $\begin{array}{l}\text { Heart rate } \\
\text { (beats/min) }\end{array}$ & $\begin{array}{l}\text { Systolic } \\
\text { pressure } \\
(\mathrm{mmHg})\end{array}$ & $\begin{array}{l}\text { Diastolic } \\
\text { pressure } \\
(\mathrm{mmHg})\end{array}$ \\
\hline First trimester & $81 \pm 11$ & $114 \pm 10$ & $72 \pm 10$ \\
Second trimester & $92 \pm 10$ & $113 \pm 8$ & $71 \pm 7$ \\
Third trimester & $92 \pm 13$ & $109 \pm 11$ & $69 \pm 10$ \\
\hline
\end{tabular}

\section{B. Radial pulse waveform characteristics difference between the three trimesters}

Fig.2 shows the means and SDs of the total pulse area, radial pulse area1, and radial pulse area2 derived from the radial pulses at the three trimesters in normal pregnant women. At the first trimester, the total pulse area, pulse areal and pulse area2 were maximum. Their corresponding values were $43.0 \pm 4.1$ for the total pulse area, $30.8 \pm 3.1$ for pulse area1, $12.3 \pm 2.4$ for pulse area2. From the first trimester to the third trimester, it clearly shows that all the pulse areas derived from the radial pulses decreased significantly (all $p<0.05$ for two paired consecutive trimesters, except the comparison between the first and the second trimesters for pulse area1). 

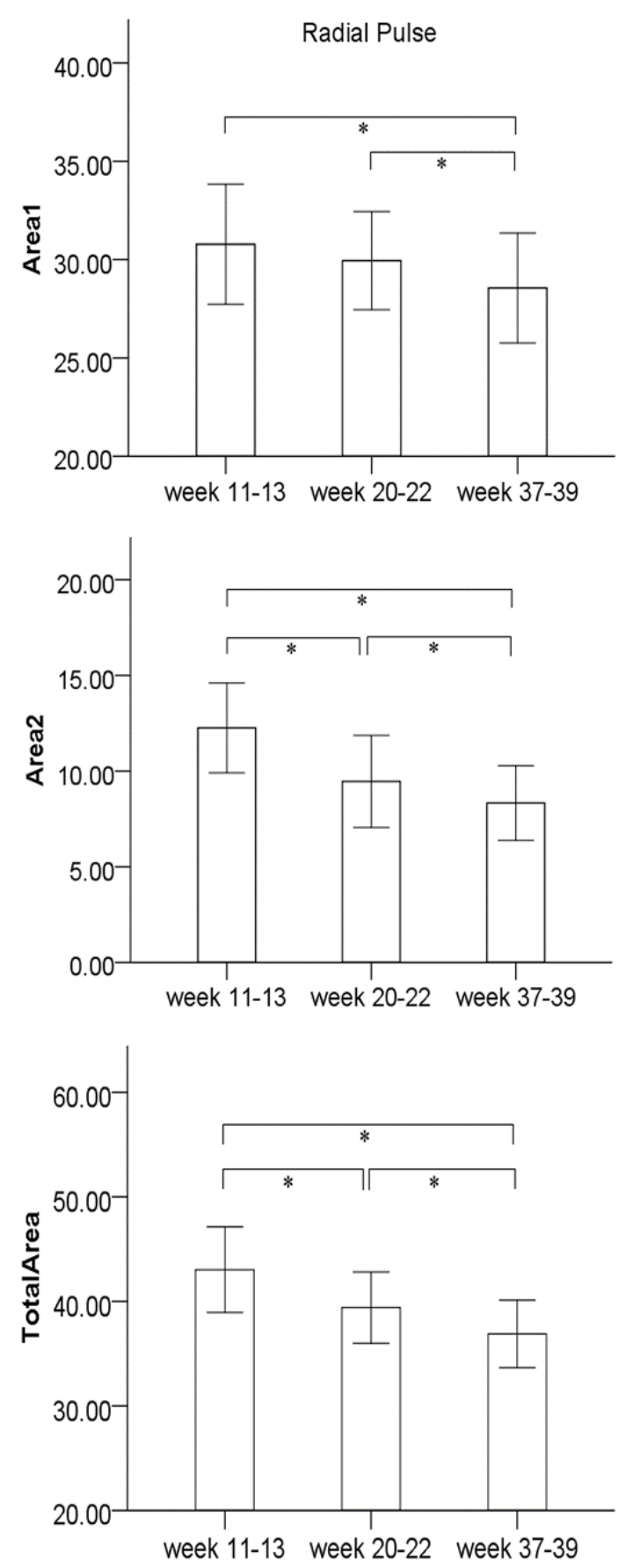

Fig.2. Comparsion of the total pulse area, pulse area1, pulse area2 of the radial pulses recorded from three different trimesters.

\section{C. $P P G$ waveform characteristics difference between the three trimesters}

Fig.3 shows the means and SDs of total pulse area, PPG pulse area1, and PPG pulse area2 derived from the PPG pulses at the three trimesters in normal pregnancy women. At the third trimester, all of PPG pulse areas values were minimum. Their corresponding values were $47.2 \pm 4.4$ for the total pulse area, $33.1 \pm 2.4$ for pulse area1, $14.1 \pm 3.5$ for pulse area2. The analysis showed that the total pulse area and pulse area 2 at the third trimester were significantly decreased in comparison with the first trimester (both $\mathrm{P}<0.05$ ).

It is also shown that area2 and total area of radial and PPG pulses had a similar changing trend, which significantly decreased with gestational trimester. However, the changes of area1 from $1^{\text {st }}$ to $3^{\text {rd }}$ trimesters performed differently between radial and PPG pulses. In detail, radial pulse areal decreased continuously, but PPG pulse area1 increased then decreased from the first trimester to third trimesters.
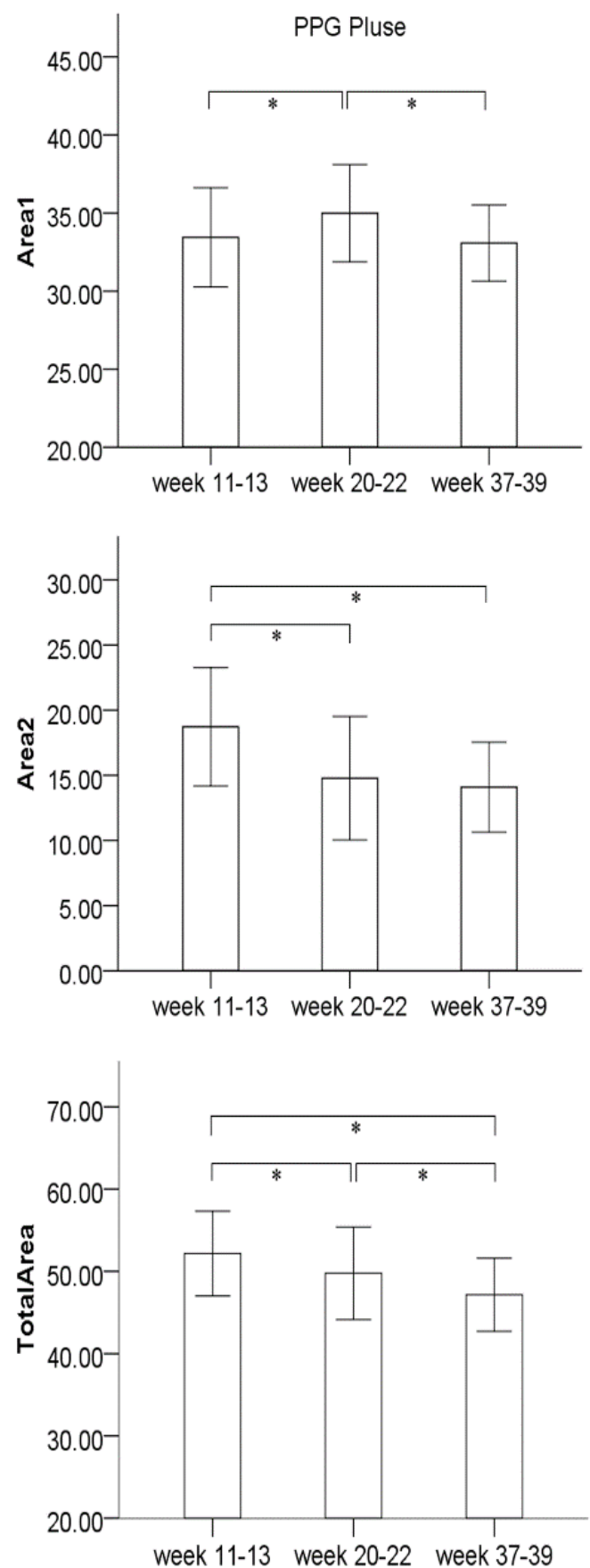

Fig.3. Comparison of total pulse area, pulse area1, pulse area2 of the PPG pulses recorded from three different trimesters. 


\section{DISCUSSION AND CONCLUSION}

In this study, the arterial pulse waveform characteristics (including the total pulse area, pulse areas before and after dicrotic notch points) derived from the normalized radial pulse waveform and the normalized PPG pulse waveform have been quantitatively and compared between the three trimesters during pregnancy.

This study demonstrated that the total pulse areas of both the radial and PPG pulses decreased gradually with gestational weeks. It has been reported that increased cardiac ejection could lead to a narrowed main wave and dicrotic wave [9]. Meanwhile, increased muscle metabolism causes muscle vasodilation and decreased muscle vascular resistance [10], resulting in lower diacritic notch point and a decreased total pulse area with gestational weeks. The physiological changes in ejection function, peripheral resistance and blood vessel elasticity during pregnancy has reflected in the total pulse area changes as demonstrated in this study [11].

Next, the decreased normalized radial pulse area1, radial pulse area2, and PPG pulse area2 have been observed in this study at the third trimester in comparison with the first trimester, expect the PPG pulse area1. It has been accepted that the pulse waveform area before the dicrotic notch point (pulse area1) mainly reflects the systolic characteristics, which is influenced by cardiac function, and the pulse waveform after the dicrotic notch point (pulse area2) mainly reflects the diastolic characteristics, which is affected by peripheral resistance and vessels compliance [12]. The increased ejection function and decreased peripheral resistance have been reported during pregnancy, resulting in the decreased pulse area 1 and pulse area 2 as observed in this study.

It is known that both the radial and PPG pulses have been widely used to evaluate cardiovascular function [13-15]. Although the physiological principle of the two pulses could be different, it has been accepted that PPG pulse and radial pulse wave are consistent in reflecting the cardiovascular function in quiet conditions [16]. In this study, it shows that these parameters derived from normalized radial pulse waveform and normalized PPG pulse waveform had similar changes trend although there was some slight differences in pulse area 1, suggesting that further investigation is required with a large number of subjects. In addition, only three measurement points were performed during pregnancy in this study, more measurements from different gestational weeks are worthy of further investigation.

In summary, this study has quantified the pulse waveform characteristic differences in terms of pulse areas between the three trimesters, providing useful scientific evidence to better understand the cardiovascular physiological changes during pregnancy.

\section{REFERENCES}

[1] P. Soma-Pillay, C. Nelson-Piercy, H. Tolppanen, et al., "Physiological changes in pregnancy," Cardiovascular Journal of Africa, vol. 27, pp. 89, 2016.

[2] Z.C Luo, "Pulse wave engineering analysis and clinical application," Science Press, 2006.

[3] D. Korpas, J. Hálek, and L. Dolezal, "Parameters describing the pulse wave," Physiological Research, vol. 58, pp. 473, 2009.

[4] G. M. London and A. P. Guerin, "Influence of arterial pulse and reflected waves on blood pressure and cardiac function," American Heart Journal, vol. 138, pp. S220-S224, 1999.

[5] J. Allen, "Photoplethysmography and its application in clinical physiological measurement," Physiological Measurement, vol. 28, pp. R1-R39. 2007

[6] J. Hashimoto and S. Ito, "Some mechanical aspects of arterial aging: physiological overview based on pulse wave analysis," Therapeutic Advances in Cardiovascular Disease, vol. 3, pp. 367-378, 2009.

[7] A. Wang, L. Yang, W. Wen, et al., "Quantification of radial arterial pulse characteristics change during exercise and recovery," The Journal of Physiological Sciences: JPS, pp. 1-8, 2016.

[8] I. Kawabata, A. Nakai, A. Sekiguchi, et al., "The effect of regular exercise training during pregnancy on postpartum brachial-ankle pulse wave velocity, a measure of arterial stiffness.(Research article)(Report)," Journal of Sports Science and Medicine, vol. 11, pp. 489, 2012.

[9] S. Munir, B. Jiang, A. Guilcher, et al., "Exercise reduces arterial pressure augmentation through vasodilation of muscular arteries in humans," American Journal of Physiology. Heart and Circulatory Physiology, vol. 294, pp. H1645, 2008.

[10] M. Nirmalan and P. M. Dark, "Broader applications of arterial pressure wave form analysis," Continuing Education in Anaesthesia Critical Care \& Pain, vol. 14, pp. 285-290, 2014.

[11] S. E. Greenwald, "Pulse pressure and arterial elasticity," QJM: Monthly Journal of the Association of Physicians, vol. 95, pp. 107-112, 2002.

[12] F. B. Reguig, "Photoplethysmogram signal analysis for detecting vital physiological parameters: An evaluating study," 2016 International Symposium on Signal, Image, Video and Communications, pp. 167-173, 2016.

[13] M. Huotari, A. Vehkaoja, K. Maatta, et al., "Arterial pulse wave analysis based on PPG and EMFi measurements," 2012 13th Biennial Baltic Electronics Conference, pp. 187-190, 2012.

[14] D.H. Nam, W.B. Lee, Y.S. Hong, et al., "Measurement of spatial pulse wave velocity by using a clip-type pulsimeter equipped with a Hall sensor and photoplethysmography," Sensors (Basel, Switzerland), vol. 13, pp. 4714, 2013.

[15] M. Crilly, C. Coch, M. Bruce, et al., "Indices of cardiovascular function derived from peripheral pulse wave analysis using radial applanation tonometry: a measurement repeatability study," Vascular Medicine, vol. 12, pp. 189-197, 2007.

[16] L. Yang, S. Zhang, and Y. Yang, "Study of Pulse Wave Velocity Noninvasive Detecting Instrument Based on Radial Artery and Finger Photoplethysmography Pulse Wave," 2008 International Symposium on Intelligent Information Technology Application Workshops, pp. 705-708,2008 